Díez, E.J. (2013). Investigación-acción participativa: el cambio cultural con la implicación de los participantes. Revista Electrónica Interuniversitaria de Formación del Profesorado, 16 (3), 115-131.

\title{
Investigación-acción participativa: el cambio cultural con la implicación de los participantes
}

\author{
Enrique Javier Díez Gutiérrez \\ Universidad de León
}

\section{Resumen}

Investigar desde una perspectiva comunitaria, desde un enfoque participativo, de compromiso y cambio con todos los agentes involucrados desde un comienzo, supone una forma de repensar la investigación desde los márgenes tradicionales de la academia.

Pero entiendo que si realmente queremos que la investigación sirva a la sociedad, a otro tipo de sociedad basada en modelos de solidaridad, democracia, justicia y bien común, debemos construir una forma de investigación coherente con el modelo de sociedad que pretendemos.

De ahí que este artículo refleje los resultados de la investigación evaluativa de carácter participativo que se desarrolló a lo largo de cuatro años en un centro docente educativo que pretendía cambiar y mejorar su organización y funcionamiento en un empeño de toda la comunidad educativa.

\section{Palabras clave}

Investigación; Investigación Acción Participativa; Investigación participativa; Compromiso.

\section{I-A-P: Cultural Change with participants implication}

Contacto:

Enrique Javier Díez Gutiérrez, enrique.diez@unileon.es, Facultad de Educación, 146. Universidad de León. Campus de Vegazana s/n, 24071-León 


\section{Abstract}

Conducting research from a community perspective, using a participatory, committed approach open to change with all those involved from the outset, implies having to relocate research beyond the traditional boundaries of academia.

However, if we really want research to be of service to society, to another kind of society based on principles of solidarity, democracy, justice and the common good, we must construct a form of research which is consistent with the model of society we are aiming for.

Hence, in this article we report the results of participatory evaluation research conducted over four years in a school, which was aimed at changing and improving its organisation and operation in an endeavour that involved the entire school community.

\section{Key words}

Research; Participatory Action Research; Participatory Research; Commitment.

\section{Introducción}

No hay cambio organizativo en profundidad, que no implique un cambio cultural, puesto que todo cambio serio afecta a las raíces mismas de la organización. "Un cambio en la organización supone un cambio de cultura, en definitiva un cambio de pensamiento de los miembros de la comunidad educativa. Se ha de plantear desde el punto actual de la organización, a través de pasos concretos con la comprensión y participación de todos" (Domenech y Viñas, 1997, 83). Este artículo describe el caso de un proceso de cambio cultural participativo y con implicación de la comunidad educativa, en un centro escolar concertado de integración de la zona de Chamartín, Madrid, que acogía alumnado no sólo con necesidades educativas específicas sino también alumnado con dificultades de convivencia y relación personal. Era un centro relativamente pequeño con un equipo de profesorado de en torno a 20 profesores y profesoras y unos 350 alumnos y alumnas. En una zona de nivel adquisitivo medio que había sido fundado en la época del franquismo por una familia con una clara vocación pedagógica.

Las preguntas que nos surgían se centraban en ¿es posible cambiar la cultura de una organización educativa? ¿Es necesario? ¿Con qué finalidad? ¿Quién ha de cambiarla? ¿Hacia dónde se debe cambiar? ¿Qué pasa con las subculturas enfrentadas? Estas y otras preguntas se hacen muchos profesionales del campo educativo cuando tienen que enfrentarse a un proceso de cambio en profundidad de la organización y funcionamiento de un centro educativo.

En un entorno cambiante y turbulento como el que nos movemos la necesidad de que las organizaciones educativas sean capaces de generar un permanente equilibrio entre la adaptación a las nuevas demandas sociales, culturales, laborales, ideológicas, etc., y la respuesta crítica ante las mismas, implica la exigencia de una permanente innovación crítica de la propia comunidad educativa. 
En un momento como el presente, en el que ya vivimos la "moda" de la calidad total, promovida por la Administración, como impulso de cambio en las organizaciones educativas, es necesario preguntarse a qué tipo de ideología responde esta propuesta de cambio. Si esta "filosofía" genera realmente una "cultura de calidad" en dichas organizaciones o si lo que puede provocar es una "cultura de la apariencia" que responda superficialmente a una imagen demandada por la administración, pero que sigue manteniendo en el fondo "lo mismo de siempre", puesto que todo cambio impuesto desde fuera no afecta a las bases de la organización.

No debemos olvidar que toda organización tiene una cultura, a menudo integrada por distintas subculturas. Y la cultura crea un entorno estable y predecible, y proporciona un sentido, una identidad y un sistema de comunicación. Una vez que el conjunto de ideas y creencias, elaborado y difundido en la organización, demuestra su funcionalidad y se consolida, puede transformarse en una "barrera invisible" a cualquier intento de cambio. $Y$ esa barrera, la cultura, se refuerza a sí misma a través de los criterios dominantes que instituye, y resulta así ser uno de los elementos más difíciles de modificar en una organización. Por tanto, todo cambio cultural, es de por sí un proceso muy lento puesto que es un elemento de contenido ideológico elaborado a lo largo de los años y que afecta a las presunciones básicas de la organización. Schein $(1990,79)$ así lo reconoce: "cuando las normas empiezan a apoyarse unas en otras, se hace necesario cambiar toda una serie de normas en lugar de cambiar sólo una o dos que pudieran estar estorbando. Por ejemplo, supongamos que un grupo desarrolló la norma de que siempre se debe buscar el consenso en las decisiones importantes y esta norma está apoyada por otras, tales como: 'se debe hablar si no se está de acuerdo con la decisión que se está tomando', 'siempre se debe decir la verdad y ser abierto en las discusiones orientadas hacia el trabajo' y 'no debe tratar de tomar la iniciativa uno mismo, a menos que el grupo haya alcanzado el consenso sobre esa acción'. Cambiar la norma para las decisiones por: 'se debe hacer lo que pida el presidente' probablemente no será posible a menos que la gente también cambie su actitud hacia la participación y su puesta en práctica".

Otros factores añadidos que dificultan el cambio cultural y que van a influir poderosamente en todo proceso de cambio son varios. El tiempo: se necesitan muchos años para lograr un cambio fundamental en la cultura de una organización. La fuerza de la cultura que se quiera cambiar: si la cultura o la subcultura anterior ha dado respuesta y sentido coherente a los miembros de la organización durante años, se sentirán renuentes a abandonarla para aceptar otra. Muchos de sus componentes se habrán convertido en elementos "sagrados" cuyo análisis, cuestionamiento y posible cambio serán muy discutidos. Lo atractivo que ese cambio les resulte a todos los miembros de la organización: cualquier propuesta con la que todo el mundo sale ganando se acepta más fácilmente que las propuestas en donde algunos tienen que perder.

Igualmente hay que tener en cuenta que la dificultad del cambio se ve incrementada porque dentro de una organización no hay sólo una cultura, sino diversas subculturas, y muchas veces en permanente conflicto: ¿cuál debería cambiar?, ¿o tendrían que cambiar todas?, ¿estarían todas de acuerdo en cambiar?

Pero esto también nos lleva a recordar que si en toda organización la cultura nunca es homogénea, y hay un permanente conflicto, la inestabilidad, la crisis y el cambio se está 
dando de hecho ya en toda organización. "La cultura nunca es totalmente aceptada ni totalmente rechazada; nunca terminan las fuerzas dominantes de instituirse y tampoco los elementos contestatarios generan el cambio radical y simultáneo de los rasgos que definen una cultura. Puede afirmarse entonces que, en la organización, sus componentes culturales están en continuo movimiento y son el resultado de una síntesis de antagonismos" (Etkin y Schvarstein, 1989, 211).

Parece, pues, que la cultura paradójicamente es un elemento dinámico (cambiante) pero de difícil cambio orientado y regulado de forma consciente e intencional. Pero lo realmente relevante es que es posible el cambio de la cultura de una organización o la reorientación de la misma. Esto se está queriendo utilizar actualmente, desde determinadas orientaciones teóricas, para sustituir la dirección a través de la normativa oficial por una dirección a través de la cultura, de tal forma que se hagan más asumibles las prescripciones diseñadas desde los cargos directivos o la Administración.

Una propuesta crítica y alternativa a ésta sólo es posible desde un proceso de cambio compartido por todos los miembros de la organización, no diseñado sólo por los directivos y "asumido" por los demás. Una alternativa que se configura desde presupuestos democráticos y de colaboración colectiva.

Por eso me centro en describir en este artículo no una declaración de intenciones, sino la articulación concreta y pormenorizada de esta alternativa metodológica, de esta estrategia de cambio cultural.

Denominé la propuesta "investigación evaluativa participativa" (Cubero, Rodríguez y Quesada, 2013), situándola dentro de la corriente de investigación-acción participativa, pero con un claro matiz evaluador. No evaluar en el sentido de medir o calificar, sino de conocer, explicitar, analizar y desentrañar entre todos los implicados aquello que acontece, aquello que conforma nuestro pensamiento y nuestra forma de hacer. Pero no sólo nos limitamos a analizar, a describir lo que ahí sucede; sino que hemos querido dar un paso más, valorar hasta qué punto esa cultura y subculturas que conforman nuestra realidad organizativa son las que queremos, las deseables; y hasta qué punto estamos dispuestos a cambiarlas.

Hacer explícitas las subculturas de la organización a través de este proceso de investigación-evaluativa-participativa, implicando a todos los sectores de la comunidad educativa en ese análisis, supone "hacer salir a la luz", explicitar, las presunciones básicas que marcan el carácter, la ideología, el funcionamiento real de esta organización. Lo cual supone establecer el aquí y ahora de la organización educativa, la cultura real que se practica día a día y que está marcando los estilos y las prácticas educativas, las relaciones y los roles de los miembros y coaliciones componentes de la organización. Esta explicitación de la cultura real, permitirá saber los logros y los problemas que se tienen respecto a la consecución de las metas marcadas en esa "cultura ideal" que deseamos, y por tanto nos ayudará a establecer, entre todos, los medios más adecuados e idóneos para avanzar en ese proyecto conjunto. En definitiva, esa evaluación de la cultura pretende mejorar la práctica y el funcionamiento del propio Centro, no sólo porque se valore el actual funcionamiento para mejorarlo, sino porque el propio proceso de valoración conlleva una dinámica cultural de acercamiento, comunicación y negociación de códigos, normas, ritos, etc., que suponen una potenciación de una cultura común y compartida. 
$Y$ en ese proceso de cambio, los propios protagonistas cambian. Se genera una cultura de solidaridad compartida, de apoyo mutuo y colaboración estrecha. Esto es lo que se ha vivido también en el propio centro donde se ha desarrollado esta experiencia. Una experiencia que condensa la capacidad de un grupo humano, de una comunidad educativa cuando se embarca en un proyecto común y compartido desde sus inicios. No diseñado por otros, ni impuesto "desde arriba", sino un proceso de cambio surgido desde el propio deseo y necesidad ante un reto que se quiere afrontar: cómo educar en una sociedad como la actual y en los momentos presentes, urgidos por una reforma educativa. Una estrategia de cambio lento y progresivo, pero en una dirección común y compartida. Deja también espacio para las disidencias, para los herejes que son capaces de seguir analizando el propio proceso desde una perspectiva escéptica o crítica. Lo cual sigue suponiendo un reto: no "dormirse en los laureles" y mostrar y demostrar que aún sigue teniendo sentido ese proceso cuando ya estamos muy lejos de sus comienzos.

Es, por lo tanto, este caso el ejemplo real de un cambio cultural en acción. El proceso desarrollado en un centro educativo a lo largo de 4 años y tres cursos escolares. Surgido inicialmente como investigación etnográfica, cómo se transforma progresivamente en un proceso colectivo, en una investigación evaluativa participativa (IEP) que permite un cambio cultural consensuado (con sus límites, por supuesto) entre buena parte de los diferentes sectores de la comunidad educativa.

\section{La culltura organizativa}

La cultura es un fenómeno complejo y difícil de definir. Y no porque sea difícil captar su existencia como fenómeno real. Todos parecemos admitir su existencia y su influencia efectiva. De la misma forma que un pez, en una fábula animada, no se percataría de vivir inmerso en un ambiente diferente al resto de las especies, los seres humanos tampoco solemos caer en la cuenta de que vivimos sumergidos en una cultura de la que somos parte y en la que nos hemos ido construyendo como personas. Tendemos a olvidar nuestro código cultural a pesar de que somos conscientes de tenerlo. De hecho para algunos autores la cultura consiste en "inadvertidos" sistemas de valores, ideas, creencias, normas, símbolos y artefactos que han sido construidos por una sociedad determinada en el transcurso de su historia y que son compartidos por las personas que la integran. "La cultura es un concepto que captura lo sutil, lo escurridizo, lo intangible, las fuerzas ampliamente inconscientes que determinan o dan forma a una sociedad, organización o grupo de trabajo. La cultura es una ficción social creada por la gente para dar significado y sentido a su trabajo y a su vida, pero a la vez es un potente y eficaz moldeador de pensamientos y conductas humanas en el interior de una organización (e incluso más allá de la organización)" (Leal Millán, 1991, 103).

La cultura parece existir en todo sistema social. Incluso podemos decir aquello de que 'toda sociedad es cultura'. Pero el problema es que no es una realidad en sí misma, sino "lo que conforma la realidad para hacerla social a través de un conjunto complejo de factores tales como los aprendizajes diversos que conciernen a la manera de ser social de los individuos y de los grupos en una sociedad determinada" (Fischer, 1992, 12). Es decir, es un constructo social que definimos aquellos que nos hallamos inmersos en ella, como "peces en el agua". 
La cultura proporciona un conjunto de soluciones y alternativas a problemas cotidianos y comunes en la vida habitual gracias a todo un conjunto de lecciones profundamente asumidas en nuestro fuero interno. Habitualmente, de manera inconsciente, todos crecemos aprendiendo qué cosas son buenas y cuáles no, qué comportamiento son aceptados y cuáles no, qué tenemos que hacer para alcanzar aquellas metas que son deseables, etc. Estas cosas se aprenden como una serie de suposiciones o asunciones asimiladas de forma natural y casi categórica. "La mayor parte de lo que nos enseña la cultura lo absorbe nuestro intestino y no nuestro cerebro. Estas cosas son tan parte de nosotros como nuestras manos o pestañas; cuando actuamos de acuerdo con ellas procedemos de forma natural, de acuerdo con una parte de nosotros mismos, así como actuamos de manera natural cuando parpadeamos. En resumen, la cultura le lava el cerebro al hombre, controla su comportamiento sin que él se dé cuenta de ello" (Litterer, 1979, 243).

Por tanto la finalidad de una cultura viene a ser interpretar, dar sentido e incluso adaptarse a su entorno. Así lo afirmaba Malinowski (1975), desde una perspectiva funcionalista, que entendía que las diferentes culturas de las sociedades y grupos humanos (ritos, costumbres, tabúes, etc.) no son sino un intento colectivo para reducir el nivel de incertidumbre y hacer el entorno más predecible y manejable. La cultura, es decir, las formas de pensar, las normas, los marcos de referencia, los valores, etc., unen a la gente en una forma natural y espontánea de enfrentarse con el medio que les rodea, de satisfacer sus miedos e interrogantes e incluso de estructurar su orden social de poder y autoridad. Pero la cultura va más allá de proporcionar códigos de conducta, conocimientos o marcos de referencia. También influye en las metas y objetivos de los individuos y los grupos humanos. Influye igualmente en el tipo de vida social que se desea y en las relaciones con los demás. En definitiva, podemos decir que la cultura moldea profundamente a la gente.

En definitiva y dicho de otra forma, podemos afirmar que la cultura sería aquel conjunto de preconcepciones, creencias inventadas, descubiertas o desarrolladas por un grupo a medida que aprende para afrontar sus problemas de adaptación externa y de integración interna, que ha funcionado suficientemente bien para ser juzgada válida $y$, consiguientemente, para ser enseñada a ("reproducido por") los nuevos miembros como el modo correcto de percibir, pensar y sentir sobre lo que sucede en la organización.

Este proceso de construcción cultural suele ser la respuesta adaptativa de la organización ante un contexto físico, ideológico y cultural o político (la cultura o subculturas actuales son las respuestas adaptativas que se consideran más adecuadas en las circunstancias presentes para el grupo que las detenta), que pasa de una generación a otra como parte de la propia historia de ella.

La cultura en este sentido posee un papel integrador que socializa los comportamientos y un papel económico que sistematiza la conducta social (no hay que reaprender permanentemente las maneras de comportarse). Así la cultura organizacional identifica a sus miembros a través de formas de pensar y de actuar en el ámbito organizacional que les son comunes y que, en consecuencia, les confieren una identidad colectiva. Esta situación le ofrece seguridad y estabilidad; pero también se convierte en un instrumento de control de los comportamientos, formas de pensar, creencias y actitudes de los miembros, que puede ser un obstáculo para el cambio y la innovación dentro y fuera de la organización. 


\section{Investigar para cambiar la cultura}

Dejando aparte el análisis transcultural comparativo, se podría afirmar que las investigaciones sobre la cultura organizacional provienen de dos sistemas metodológicos principales (Thévenet, 1992): El Desarrollo Organizacional que se ha dedicado al estudio, desde la perspectiva cultural, aplicado a las organizaciones en el marco de la ingeniería cultural (cultura como variable interna) para manejar y cambiar la cultura con el fin de mejorar el éxito y la eficacia de la organización. Y, por otra parte, el segundo modelo que deriva de un enfoque "etnográfico" de la organización.

Los enfoques etnográficos, que es la orientación por la que se ha optado en esta investigación, inciden en la diversidad y amplitud de las informaciones que se deben recoger, estudiar y verificar. Se centran más en la preocupación por perfeccionar una descripción de la organización como sistema cultural. Los factores clave de la investigación se relacionan con el discernimiento del equipo investigador, con la calidad de sus informaciones y de sus relaciones con los miembros de la organización para recoger, valorar, verificar y explicar esta materia. El esquema etnográfico considera a la organización como un sistema cultural e intenta describirla y detectar sus problemas desde esta perspectiva. Estos enfoques utilizan preferentemente las metodologías cualitativas, basadas, sobre todo, en estudios de casos y en las escalas de observación y entrevistas, entre otros instrumentos. No obstante este tipo de investigación no puede olvidar la necesidad de complementar las diferentes perspectivas a través de la triangulación de fuentes, métodos (cuantitativos y cualitativos), instrumentos, etc., como medida de validación y fiabilidad del propio proceso de investigación. Cada vez más las organizaciones se estudian siguiendo esquemas explícitamente interdisciplinares, que recogen tanto las aportaciones de la sociología, como de la psicología o de la antropología y de la teoría organizacional.

Pues bien, la propuesta que diseñamos de cara a investigar la cultura organizativa de una centro educativo, fue la evaluación entendida como proceso de investigación evaluativa de tipo holístico, participativo, de carácter fundamentalmente cualitativo, marcada por la triangulación, la negociación y el compromiso entre todos los sectores de la organización.

Esto conlleva tres finalidades:

1) Generar este proceso de evaluación participativa supone que los propios implicados puedan reflexionar sobre aquellos aspectos soterrados (currículum oculto, creencias, predisposiciones, formación anterior y estilos de funcionamiento) de la práctica educativa y del trabajo profesional que pueden estar facilitando o impidiendo una dinámica de cambio o innovación dentro del centro o institución.

2) La segunda finalidad que se persigue es que mediante este proceso de reflexión y debate colectivo se genere a su vez una cierta "cultura" de trabajo en equipo, participativa y de colaboración, que permita la continuidad de estos procesos aplicados a otros aspectos de la investigación o fases, con un alto nivel de autonomía y autogestión por parte de los miembros de un centro u organización.

3) $Y$ finalmente, que lleve a establecer una serie de acuerdos por consenso, que supongan un reparto y compromiso de acciones y tareas concretas para todos los miembros de la comunidad educativa en cada una de las fases del proceso de innovación y 
cambio, en los cuales definieran su propio concepto de cultura de calidad en la educación y decidan las estrategias a desarrollar para alcanzarlo.

Este planteamiento no es una estrategia cerrada, simple y lineal, que pueda diseñarse previamente desde principio a fin. Es un proceso que debe replantearse continuamente, según vayan apareciendo nuevos datos y de acuerdo a las circunstancias que se vayan produciendo (normas externas de la Administración, decisiones que implican despidos o reajustes jerárquicos de la plantilla, el tiempo o las nuevas directrices políticas, entre otros aspectos o circunstancias que suelen aparecer en procesos con una duración de un curso escolar o más). Hay que tener en cuenta que es un proceso de reflexión conjunta de los profesores o formadores y de toda la comunidad u organización e institución (agentes externos e internos) sobre su propia realidad y experiencia, para articular un proyecto común.

Desarrollar todos estos procesos supone en alguna de las fases reflexionar sobre esos supuestos (creencias, valores, predisposiciones, formación previa, situaciones y circunstancias concretas) del grupo implicado en el proceso de investigación, que no es otra cosa que evaluar la cultura y las subculturas que configuran el centro o institución. Porque en este tipo de investigación participativa y democrática no sirve el diseño metodológico y teórico decidido por el equipo de investigación, si no es negociado y consensuado con los participantes de la propia investigación. Esto es la opción elegida cuando se propuso llevar a cabo un nuevo modelo de investigación evaluativa: la investigación evaluativa participativa.

La investigación evaluativa se entiende siempre que se da un proceso de reflexión crítica y sistemática sobre los hechos y actuaciones sociales con una finalidad de aportar datos, hechos y elementos de juicio que posibiliten la toma de decisiones racionalmente fundamentadas, que se siente comprometida con la sociedad en general y con el desarrollo y profundización de la democracia política y que concibe a los miembros de la organización como investigadores. Y esto viene a caracterizar la investigación evaluativa distinguiéndola de otras formas de investigar en las que el investigador se mantenía alejado de los fenómenos, escudado en una actitud de no ingerencia y de no intervención que asegurasen la supuesta posibilidad de una objetividad "pura". Supera así la dicotomía sujeto-objeto en el proceso de investigación, haciendo recaer el peso de la objetividad en la experiencia compartida, por lo cual, el objeto es fruto de la intersubjetividad social. Desde esta perspectiva cualitativa, cuando se investiga se evalúa, puesto que el conocimiento no es sólo para incrementar el patrimonio de la ciencia, sino que su finalidad fundamental es mejorar la acción desarrollada hasta ahora, generando un proceso de retroalimentación y entendimiento de lo que se ha hecho para hacerlo mejor en un futuro. Pues bien, dentro de este enfoque de carácter eminentemente cualitativo, la evaluación que se propuso tiene un sesgo particular: es una "investigación evaluativa de carácter participativo".

Esto supone adentrarse en los presupuestos y postulados de la investigación-acción participativa. Frente a la investigación orientada hacia la búsqueda de conocimiento, la investigación propuesta busca información para tomar decisiones en orden a la intervención social -investigación evaluativa-, es decir un proceso de comprensión y explicación realizado por los propios miembros que intervienen en el programa sobre su práctica con el fin de mejorarla. $Y$ esta perspectiva, por consiguiente, no se fija tanto en 
cómo se accede al conocimiento, sino para qué se busca dicho conocimiento, subordinando así las cuestiones de carácter metodológico a los objetivos y fines que se pretenden alcanzar. En este sentido, para la teoría crítica no existe distinción entre teoría y práctica, ya que es la finalidad de la investigación la que, en última instancia, aporta la credibilidad teórica a un proceso de investigación. Y la investigación acción es la estrategia metodológica apropiada para llevar a cabo este enfoque.

Este tipo de investigación evaluativa participativa supone:

Un carácter democrático: los problemas son definidos por todos los afectados (no sólo por los profesionales o los investigadores), se recogen los datos, se interpretan y valoran los resultados por parte de todos y todas, etc. En definitiva, participan y se implican todos, asumiendo el control del proceso y de la toma de decisiones. Es un proceso de aprendizaje de tolerancia, participación y toma de decisiones democráticas para los propios implicados. Pero también es un proceso de formación en la investigación pues hay que dotar de herramientas y comprensión a la comunidad que se involucra en el proceso de análisis de su propia realidad. De esta forma se asume una visión democrática del conocimiento y de los procesos educativos implicados en su desarrollo, que incluso conlleva una opción por los más oprimidos.

Un carácter educativo de la experiencia investigadora: es un proceso colectivo que conlleva una experiencia educativa. No sólo porque participar implica ejercitar los valores cívicos del ejercicio democrático, sino porque supone igualmente adquirir conocimiento más objetivo de la organización, de su cultura, aprender a analizar conjuntamente con más precisión sus problemas, descubrir los recursos de que disponemos, generar un lenguaje compartido, confrontar y debatir los enfoques y las visiones que aparecen, formular las acciones pertinentes para mejorar. Investigación y educación se consideran como momentos de un mismo proceso, es decir, la investigación participativa se transforma en quehacer de aprendizaje colectivo. Pretende así favorecer procesos de análisis y de diálogo compartido a partir de la propia experiencia que tenga potencial educativo, de reflexión, cambio y mejora para todas las personas implicadas.

Le confiere un carácter de investigación-acción al proceso de investigación evaluativa: entre la investigación evaluativa y la acción educativa se va dando así una interacción permanente. La reflexión evaluativa sobre la práctica educativa cotidiana se convierte en fuente permanente de información sobre el acierto o error de las mejoras introducidas. Convertimos así el proceso evaluativo en una dinámica inscrita en el mismo corazón del proceso educativo, y el proceso educativo en un proceso continuo y permanente de investigación-acción real y efectivo.

Un carácter participativo y de implicación de la comunidad: entre los participantes en la investigación se crea una situación de interacción activa, de diálogo y negociación. Y no sólo por la participación de los distintos sectores, sino que también hay que tener en cuenta que a todas las personas implicadas se las ve como a iguales, puesto que no se busca la "verdad", sino conocer la perspectiva de los otros y las otras. Es un tipo de investigación evaluativa que busca ponerse al servicio de los grupos sociales y de las comunidades.

Obviamente, la participación en el proceso de investigación evaluativa requiere preparación y seguimiento. Implica establecer una dinámica evaluativa en clara correspondencia con un proceso formativo del grupo, estimulando progresivamente la capacidad evaluadora de los 
participantes a fin de que ellos lleguen a apropiarse no sólo de la realización sin de la dirección del proceso. Para que progresivamente la figura del equipo de asesoresevaluadores externos vaya desapareciendo, se termine determinando su "no necesidad", y la propia comunidad educativa declare su "autonomía evaluativa".

La finalidad de este tipo de investigación evaluativa de carácter participativo no sólo busca mejorar la práctica educativa y el funcionamiento de la organización, sino que da un paso más allá. Persigue igualmente la transformación de la realidad social y la promoción de un desarrollo comunitario basado en el protagonismo de todos los sectores implicados. Trata de crear en los participantes autoconciencia de su realidad social y desarrollar en ellos capacidad para tomar decisiones de cara a mejorarla. En definitiva persigue concienciar, activar a los participantes, capacitar a la gente para movilizar sus propios recursos humanos y materiales para la solución de los problemas sociales. Que sean ellos los protagonistas de su propio cambio, de su propio avance.

La participación y la implicación de la comunidad educativa en la dinámica organizacional se ha enfocado desde muchas perspectivas y la literatura sobre el tema es muy abundante en todas ellas. Pero, "lo que sucede con la participación es que no es sólo una técnica o una estructura concreta organizativa, sino que es una cultura” (Pascual, 1996, 41). La Ley Orgánica del Derecho a la Educación de 1985 propuso la posibilidad y funcionamiento de una nueva cultura en la vida de los centros educativos, propuso unas nuevas estructuras participativas que presuponían una cultura para la que tal vez no estuvieran preparados dichos centros. La disonancia estructura-cultura participativa es fuente de innumerables dificultades que se experimentan en la vida escolar: desilusión, falta de seriedad, inhibición, ineficacia. No se puede pensar que por el hecho de introducir una estructura de participación se consigue sin más el cambio.

Una tarea importante si se quiere formar auténticamente una comunidad educativa es crear una cultura propia, que debe buscarse y fomentarse como tal, por todos los miembros de la comunidad. "En realidad, la importancia concedida en los últimos años a la cultura organizativa de los centros escolares ha hecho resaltar la concepción de la escuela como comunidad frente a la escuela como organización formal” (Coronel et al., 1994, 107)

Pues bien, la aplicación práctica de esta investigación no ha sido sino el intento de potenciar una cultura de participación e implicación a través de un proceso concreto de investigaciónacción en el que en el mismo proceso de evaluación diagnóstica y conocimiento de la cultura organizativa del centro se ha potenciado una cultura de consenso, una cultura de acercamiento entre las distintas subculturas. La pretensión en la que se embarcó la comunidad educativa de este centro fue crear un centro con cultura participativa donde las normas y los valores democráticos fueran ampliamente compartidos, se expresaran en sus documentos y guiaran la conducta de sus componentes, agotando las posibilidades de consenso en los procesos decisionales.

De esta forma se trataba de evitar un planteamiento de ingeniería cultural o "management simbólico", orientando el cambio cultural desde una perspectiva democrática, participativa, colaborativa e implicativa en cuyo proceso tomaran parte activamente todos los sectores implicados (aunque es necesario reconocer el "fracaso" relativo en la implicación de una buena parte del sector de familias dado que el profesorado no supo o, en algunos casos, era reticente a implicarlas y también por la falta de tradición de participación de muchas 
familias, así como de los tiempos que se planteaban para realizar el proceso que solían ser horarios donde algunas familias aún estaban trabajando). El cambio compartido ha de ser un cambio promovido desde dentro a partir de las propias inquietudes y necesidades. Es también un cambio que debe partir del análisis de la cultura existente, del estudio de las razones que llevan a su instalación y a su cambio y de la selección de las estrategias más adecuadas a un determinado contexto.

El proceso ha sido lento, comenzando desde una perspectiva práctica en torno a la investigación, análisis y evaluación de las prácticas docentes cotidianas, de cara a motivar al profesorado al percibir de una forma casi inmediata y rápida la utilidad de investigar y reflexionar sobre su propia práctica. Entendimos que la democratización del proceso de investigación debía pasar inicialmente por analizar, conjuntamente con el profesorado, el modo en que estructural y culturalmente se regulan las prácticas del trabajo docente. Por ello el primer paso fue conocer y profundizar en las prácticas docentes, para avanzar hacia las prácticas organizativas y de ahí al trasfondo cultural de las mismas.

El proceso fue tan positivo que se generó una dinámica permanente de innovación y cambio, así como de investigación-reflexión continuada, que ya no necesitaba de expertos o asesores externos que potenciaran esa dinámica. Empezaron a surgir procesos de investigación diversos, al margen del propio equipo de investigación. Así, el análisis y evaluación de las formas y prácticas de evaluación que se llevan a cabo en el centro, para establecer criterios comunes y acordes con el Proyecto Educativo de Centro consensuado entre todos, es un proceso de investigación-reflexión que no ha surgido del equipo investigador, aunque participa y colabora en él. Por lo que puede verse que esta dinámica se ha inscrito en la médula del funcionamiento organizativo del centro, generando una cultura de compromiso y coordinación en la práctica educativa real, autónoma y con continuidad desde las propias necesidades y definiciones de la realidad del centro.

\section{Conclusiones, implicaciones y discusión sobre el proceso de cambio cultural}

Los resultados de esta investigación se han centrado no sólo en describir el contenido de la cultura de la organización, sino también su origen, su evolución y su estado actual, así como la orientación futura que está tomando en base a ese proceso de unificación cultural desencadenado.

Hay que entender que los resultados obtenidos en un tipo de investigación como ésta no tratan de ser universalizables, sino útiles para mejorar la práctica educativa de la propia organización, y, en todo caso, que puedan ser transferidos, contextualizándolos, aquellos aspectos significativos que pueden ser aplicables y útiles a otras organizaciones educativas en similares situaciones. Por eso esta exploración ha tratado de comprender en profundidad los significados de la red de relaciones que tejen las actitudes y conductas de los protagonistas de la acción escolar desde una perspectiva etnográfica: la acción puede ser entendida sólo en el sentido de cómo la gente concernida concibe su situación a partir de la observación participante; las entrevistas en profundidad serían las llaves del significado con vista a explorar las acciones individuales y a clarificar las intenciones, percepciones e interpretaciones de las situaciones estudiadas; pero el significado de las 
acciones también pueden ser estudiadas examinando documentos que el centro elabora mediante el análisis documental.

En definitiva se ha desencadenado un proceso de innovación con una carga futura de mejora progresiva, pues se basa en la participación real de la comunidad educativa en la propia dinámica del centro. Innovación, no como resultado de una ingeniería social programada, basada en una racionalidad técnica: transmisión unidireccional, expertos que deciden desde arriba, etc. Sino un proceso de innovación como indagación colectiva mediante el diálogo, la reflexión y el análisis crítico. Como dice Imbernón (1995, 69) "la innovación educativa sólo será posible si los profesores y las profesoras son capaces de adoptar compromisos, actitudes y propuestas investigadoras en sus clases y centros, pues todo proyecto innovador en educación ha de ser paralelo al desarrollo del profesorado y al de la investigación desde la práctica. Así pues, la innovación educativa aparece como proceso de investigación impulsado desde la base (...). Por tanto, se introduce el concepto de que la innovación para el cambio educativo supondrá entender éste como un proceso de investigación colectiva en el que todos los elementos educativos se implican". Implicación que ha de ir asumiendo progresivamente al resto de la comunidad educativa, pues no podemos quedarnos en propuestas de innovación técnicas, a cargo exclusivamente de los "profesionales de la educación". La labor educativa está abocada al fracaso si no consigue implicar a todos los sectores afectados en una dinámica convergente, en la construcción de una cultura compartida.

Y creo que en este proyecto de investigación se consiguió. El profesorado se implicó progresivamente, prescindiendo del "andamiaje" de un equipo investigador externo que apoye y dinamice el proceso, generando una estructura estable y autónoma de investigación-acción sobre la propia realidad del centro. El equipo directivo, no sólo participó, sino que animó y apoyó el proceso de una forma clara y directa. El alumnado participó progresivamente también, a través de los claustros de evaluación, a través de la evaluación al propio profesorado y a la dinámica del centro, incluso, los mayores llegaron a participar en el diseño de la investigación, la recogida de información y su posterior análisis como se ha expuesto a lo largo de la investigación. $\mathrm{Y}$ finalmente, las familias han participado, aunque minoritariamente, pero con aportaciones claras y contundentes.

Porque esto era lo que en definitiva se perseguía en esta investigación, servir a la mejora educativa en el centro: que los integrantes de la comunidad educativa pudieran conocer su cultura e implicarles en este análisis en profundidad, para que desde ese conocer pudieran transformar su propia realidad de una forma consciente y planificada hacia unas metas explicitadas y consensuadas por todos.

Se han cumplido los objetivos más importantes de esta investigación: generar un proceso de cambio e innovación y, a la vez, implicar en él a toda la comunidad educativa, mediante el cual se potenciara una cultura de participación y compromiso. Y no un cambio que se quedara en la epidermis de la pedagogía del aula, sino alcanzando las estructuras de la organización, las relaciones sociales, las rutinas incuestionables instaladas en la escuela. Pero también, que este proceso no se quedara aquí, sino que se orientara a su consolidación e institucionalización como un proceso permanente de innovación de forma autónoma e independiente. 
"La innovación, entendida como investigación en la práctica, necesita de nuevas y viejas reivindicaciones y de una nueva cultura profesional que se ha de forjar en los valores de la colaboración y del progreso social, entendido esto como transformación. (...) La posibilidad de innovación en el campo del currículo en los centros no puede plantearse seriamente sin un nuevo concepto de profesionalización del profesorado en esa cultura (...). La innovación ha de ser intrínseca al proceso educativo y profesional, hay que establecer mecanismos laborales y estructurales para facilitarla conjuntamente con el cambio cultural de la profesión" (Imbernón, 1995, 71).

Como se ha podido comprobar en esta investigación, el cambio no puede ser total, radical ni inmediato. No es realista proponer un cambio total de estilo, actividades y modos de hacer en una organización educativa, porque no resulta posible: una persona dedicada a la docencia ha ido elaborando un sistema de trabajo que comprende y domina, en el que se encuentra segura. Pretender que esta persona se cuestione, se exponga a la crítica y cambie de forma rotunda lo que hace, es muy dificultoso. Porque la cultura es fuente de seguridad, de conocimiento en que así las cosas han funcionado hasta ahora, y todo cambio supone un cálculo de riesgo, al que no todo el mundo está dispuesto a asumir de forma radical. Por esto sólo es posible un cambio progresivo, partiendo de experiencias concretas, cotidianas y que supongan un éxito a corto plazo y palpable. Un cambio en el que se embarque un colectivo, compartido y asumido por todas las personas implicadas, que provoque una alternativa de seguridad compartida, pues el miedo a equivocarse sólo, es un factor de riesgo que aumenta la prevención frente al cambio.

De ahí que planteemos que utilizar la cultura, desde un enfoque de "ingeniería cultural" (Fierro y otros, 2013), para manejar de una forma más sofisticada el funcionamiento y la organización de un centro es una perspectiva equivocada y antidemocrática.

Desde la perspectiva de la nueva sociología de la educación se considera que esta perspectiva "ingenieril", debido al legado teórico estructural-funcional, aunque pretenda ser neutral y objetiva, tiende a ignorar o encubrir unos intereses esencialmente ideológicos al ocultar conflictos inherentes y rasgos estructurales importantes de la organización, particularmente la estructura de poder y conflicto, la desigualdad estructurada y la significación de la estructura de 'culturas de clase'. Lo que se cuestiona es si, en el fondo, lo que se pretende con la ingeniería cultural no sea sino conseguir un 'control ideológico' en las organizaciones educativas, en lugar del anterior control burocrático, un instrumento sutil para hacer más eficiente y aceptables las prescripciones oficiales determinadas desde los niveles de poder central. Un control basado en "manipular" la cultura organizativa. De hecho todo el discurso actual sobre la eficacia escolar y la cultura escolar 'excelente' originado al trasladar un discurso surgido en el ámbito industrial a la escuela- remite a una manipulación cultural en aras de buscar dicha excelencia. $Y$ una cosa es la preocupación antropológica por entender, y otra bien distinta la preocupación gerencial por manipular.

Desde esta perspectiva habría preguntarse: quién o quiénes son los que definen la excelencia organizacional que se persigue con la tan traída y llevada eficacia organizativa. Parece que hasta ahora se decanta el asunto por los directivos. En este sentido entonces, nos podríamos preguntar igualmente: ¿excelencia para quién?, ¿según la entienden los profesores/as, los alumnos/as o la dirección?; ¿eficacia a costa de quién?, ¿a costa de qué? De 
quien depende realmente el éxito o el fracaso de un proceso de cambio es del conjunto del profesorado y del resto de la comunidad educativa. Núcleos directivos técnicamente eficaces o expertos en Proyectos de Centro no garantizan un proceso de cambio colectivo. La insistencia de las Administraciones en nombrar a los equipos directivos es un desenfoque de las necesidades del sistema educativo, además de poner de manifiesto la creencia en que el cambio proviene básicamente de la aplicación de técnicas de gestión.

La defensa contra esta manipulación soterrada e interesada de la "cultura organizacional” y la propuesta de una "cultura compartida" desde su definición participativa y negociada, ha sido la intención de esta investigación y la utilidad en definitiva para los propios integrantes de la organización que han "sufrido y vivido" este proceso de investigación-acción permanente y nunca acabado. La meta está en el propio camino, puesto que en el quehacer diario es donde se fragua esa cultura compartida. La explicitación de la misma; su análisis en profundidad; el debate, negociación y consenso que conlleva sobre las preconcepciones que tienen las distintas coaliciones y subculturas respecto al ser humano, al mundo, a la realidad, la educación, etc.,..., ahí es donde se va consolidando progresivamente una cultura compartida y asumida por todos. No impuesta desde una dirección eficaz, sino decidida colectivamente.

En definitiva, es afirmar que el proceso de investigación evaluativa participativa de la cultura de un centro puede convertirse en un instrumento para la mejora del funcionamiento interno del mismo y de su calidad educativa como tal. Un proceso de investigación evaluativa que fomenta los procesos participativos de formación y reflexión, convierte este proceso de autorreflexión y autoevaluación de la actividad del grupo en un instrumento interno para su mejora profesional y de la satisfacción respecto a sus expectativas de la comunidad educativa, potenciando así una cultura de consenso y compromiso que lleva a la autorresponsabilidad del propio profesorado y del resto de la comunidad educativa (a nivel individual y grupal).

Finalmente, recordarnos que un tipo de investigación como ésta no es el final del proceso de investigación-acción en el centro educativo, sino el punto de arranque de otro nuevo comienzo en el proceso permanente de mejora de nuestra práctica profesional y de ayuda a unos seres humanos que están creciendo y que necesitan lo mejor de nosotros/as mismos/as en una labor compartida de acercamiento al mundo y a la felicidad. Generar un contexto de colaboración para el apoyo profesional mutuo; la pertenencia a un grupos de compañeros y compañeras que permita superar el aislamiento y la soledad profesional entre el profesorado; la existencia de metas comunes elaboradas y definidas en colaboración; un entorno orientado a la resolución de problemas en vez de al ocultamiento de los mismos; una estructura de incentivación basada en la implicación y la progresión profesional en vez de en recompensas externas (Escudero, 1990); todo esto, no puede sino repercutir positivamente en el proceso de enseñanza y aprendizaje que es, al fin y al cabo, la meta de toda organización educativa.

La última reflexión que quiero reflejar aquí es la insistencia, una vez más, en que esta investigación se inscribe en el campo de la metodología (propone una metodología de investigación alternativa: Investigación Evaluativa Participativa), de la didáctica (desarrolla propuestas concretas de intervención didáctica en el aula y en el centro), y de la organización (se adentra en el fenómeno de la cultura organizativa de una institución). Pero 
destacar igualmente que se ha planteado también desde un enfoque preventivo. Porque un campo privilegiado de prevención es el que se sitúa en el "escalón inicial del trayecto del fracaso escolar-social-personal” en los centros de educación. Consideramos que tan sólo abordando adecuada y flexiblemente la normalidad podemos atender la dificultad. Se trataba de atender no al alumno diferente o con dificultades, sino abordar el cambio y la mejora de una forma global, desde el enfoque del grupo-clase como diversidad compleja de la que partir. Y para ello es necesario tener en cuenta la cultura de centro, en qué medida potencia o rehúye prevenir, integrar, atender a aquellos con una situación de riesgo más extrema: "el sentido de una actividad o estrategia compensadora o especial depende no tanto de sus componentes técnicos y organizativos como del valor y significado que tienen en el contexto de centro: si el proyecto y la cultura de centro emiten un mensaje integrador y promocionador o si emiten un mensaje jerarquizador y clasificador" (Muñoz, 1995, 68).

Esta investigación no se ha preocupado tanto por obtener información científica, cuanto que esa información sirviera para solucionar problemas concretos y mejorar la práctica del propio centro. Por eso uno de los criterios fundamentales de cientificidad -puesto que reivindico la no exclusión de los fines del pensamiento científico- es el para qué de la investigación, la finalidad de los conocimientos adquiridos. Y si evaluamos la repercusión social de esta investigación evaluativa que se ha llevado a cabo a lo largo de estos tres años en el centro, creo que ha sido útil socialmente el proceso, los datos obtenidos, el conocimiento alcanzado y las transformaciones habidas para toda la comunidad educativa implicada generando una unificación cultural progresiva y una dinámica de autoevaluación, innovación y cambio que se está notando cada vez más.

\section{Bibliografía}

Coronel, J.M, López, J. y Sánchez, M (1994). Para comprender las organizaciones escolares. Ocho temas básicos. Sevilla: Repiso Libros.

Cubero, J., Rodríguez, G. y Quesada, V. (2013). Investigación e Innovación Educativa al Servicio de Instituciones y Comunidades Globales, Plurales y Diversas. En María Cristina Cardona Moltó, Esther Chiner Sanz, Antonio Giner Gomis. Actas del XVI Congreso Nacional / II Internacional Modelos de Investigación Educativa de la Asociación Interuniversitaria de Investigación Pedagógica (AIDIPE), págs. 828-836. Alicante, 4-6 de septiembre.

Domenech, J. y Viñas, J. (1997). La organización del espacio y del tiempo en el centro educativo. Barcelona: Grao.

Escudero, J.M. (1990). El pensamiento del profesor y la innovación. En Villar, L.M. (Ed.) Pensamiento de los profesores y toma de decisiones. Sevilla: Universidad de Sevilla.

Etkin, J. y Schvarstein, L. (1989). Identidad de las organizaciones. Invariancia y cambio. Barcelona: Paidós.

Fierro, E., Mercado, P. y Cernas, D.A. (2013). El efecto de la cultura centrada en el conocimiento y la interacción social en la innovación organizativa: el efecto mediador de la gestión del conocimiento. Esic market, 145, 87-108 
Fischer, G. N. (1992). Campos de intervención en Psicologia Social. Grupo, institución, cultura, ambiente social. Madrid: Narcea.

Imbernón, F. (1995). Innovación y formación en y para los centros. Cuadernos de Pedagogía, 240, 68-72.

Leal Millán, A. (1991). Conocer la cultura de las organizaciones: una base para la estrategia y el cambio. Madrid: Actualidad.

Litterer, J. (1979). Análisis de las organizaciones. México: Limusa

Malinowski, B. (1975). Una teoría científica de la cultura. Barna: Edhasa.

Muñoz, E. (1995). La respuesta democrática. Cuadernos de Pedagogía, 238, 64-69.

Pascual, P.R. (1996). La función directiva en el contexto socio-educativo actual. En R. Pascual (Coord.). La gestión educativa ante la innovación y el cambio (37-51). Madrid: Narcea.

Thévenet, M. (1992). Auditoría de la cultura empresarial. Madrid: Díaz de Santos. (Original: (1986). Audit de la culture d'enterprise. París: Les Éditions d'Organisation).

Schein, E.H. (1990). Consultoría de procesos. México: Addison-Wesley y Sistemas Técnicos de Edición. 


\section{Autor}

Enrique Javier Díez Gutiérrez

Facultad de Educación Despacho 146. Universidad de León. 24071 León

Tfno: 987-291437 Fax: 987-291135; enrique.diez@unileon.es

Profesor Titular de Universidad en la Universidad de León. Participa en colectivos universitarios como el Grupo Acoge (Educación, inclusión e igualdad) o el movimiento "Otra investigación es posible". En movimientos sociales como el Foro por la Memoria de León, el Grupo de Hombres por la Igualdad o Hombres por la abolición de la prostitución. Entre sus publicaciones se encuentran: Educación Pública: de tod@s, para tod@s (Bomarzo, 2013), Qué hacemos con la educación (Akal, 2012), Educación Intercultural: Manual de Grado (Aljibe, 2012), "Decrecimiento y educación" con Carlos Taibo (Catarata, 2011), o Globalización neoliberal y sus repercusiones en la educación (El Roure, 2007) 